\title{
Morphology dependent biological behavior of calcite materials
}

\author{
Hyoung-Mi KIM, Mi-Kyung KIM, ${ }^{*}$ Jong-Young LEE, Jong Kwon LEE, ${ }^{* *}$ \\ Jayoung JEONG, ${ }^{* *}$ Soo-Jin CHOI ${ }^{*, \dagger}$ and Jae-Min OH ${ }^{\dagger}$ \\ Department of Chemistry and Medical Chemistry, College of Science and Technology, Yonsei University, \\ Wonju, Gangwon-do, 220-710, Republic of Korea \\ *Department of Food Science and Technology, Seoul Women's University, \\ 621 Hwarang-ro, Nowon-gu, Seoul 139-774, Republic of Korea \\ ** Toxicological Research Division, Ministry of Food and Drug Safety, \\ Osong-eup, Chungchungbuk-do 363-700, Republic of Korea
}

\begin{abstract}
We have evaluated the morphology dependent biological behaviors of calcite particles in simulated body fluid conditions. Two kinds of calcite particles with different morphologies (cubic and spicule) were prepared utilizing different polyol solvents. According to X-ray diffraction, scanning electron microscopy, zeta potential measurement and dynamic light scattering study, two calcite samples had different morphology but other physicochemical parameters like particle size and surface chemistry of two samples were similar. The biological behaviors of two calcites were evaluated in simulated gastric and intestinal condition considering the oral administration of calcite as potential bio-medical applications. Calcium dissolution rate, change in zeta potential and interaction with proteins of differently shaped calcites in simulated biological conditions were evaluated. Spicule shaped calcites showed facilitated surface calcium ion dissolution compared with cubic shaped one in simulated gastric condition. The zeta potential measurement and protein fluorescence quenching assay results revealed that the spicule calcites interacted more actively with biological substances like electrolytes and proteins than cubic shaped one. Scanning electron microscopic images of calcites treated in simulated body fluid exhibited that the biological behaviors of calcites could be mainly determined by the dissolution and particle-biosubstance interaction at the surface of calcite particles.
\end{abstract}

(O2014 The Ceramic Society of Japan. All rights reserved.

Key-words : Calcite, Morphology, Biological behavior, Simulated body fluid, Surface interaction

[Received February 15, 2014; Accepted April 13, 2014]

\section{Introduction}

Calcite, one of the most stable polymorph of calcium carbonates, has been extensively studied in both academic and industrial fields due to their versatile applications as soil remediating agents, spacing extender for pigments, fillers in rubbers, concrete repairing agents and etc. ${ }^{1), 2)}$ Calcites obtained from sea shells or oysters are medicinally utilized as calcium supplements or as antacid. ${ }^{3)}$ Due to the potential applicability of calcites in bio-medical fields, there have been increasing interests on the relationship between physico-chemical properties of calcite and bio-medical activities. Many researchers reported that the physico-chemical parameters of calcite including crystal phase, crystallinity and particle size could influence their dissolution in physiological condition or bioavailability. ${ }^{4)}$ For example, calcite powder ground in nano-scale was reported to exhibit different biological behavior compared with bulk calcite powder. Thus food grade nano-calcite is considered as effective calcium supplement and several products utilizing nano-calcite such as Nano-Cal ${ }^{\mathrm{TM}}$ (bionetics, America) and Nano-Ionic Calcium \& Magnesium $^{\mathrm{TM}}$ (MMSAustalia, Australia) are now commercially available.

Like this, it has been generally reported that materials, having same chemical composition, interact differently with biological

\footnotetext{
Corresponding author: J. M. Oh; E-mail: jaemin.oh@yonsei. ac.kr

\# Corresponding author: S. J. Choi; E-mail: sjchoi@swu.ac.kr

tit Preface for this article: Dol http://dx.doi.org/10.2109/jcersj2.122.P8-1
}

substances according to their physico-chemical parameters like particle size, shape and surface chemistry. Researches on the relationship between physico-chemical parameters and biological behaviors have been mainly conducted on nanomaterials, as recent progresses in nanotechnology ignited the development of bio-medically applicable nanomaterials. The most well comprehended parameter is size of materials; as size decreases, the specific surface area increases consequently resulting in active interaction between materials and biological substances. ${ }^{5)}$ Surface chemical properties like surface charge was also known to play a key role in biological interaction; when materials have countercharge with biological substances such as electrolytes, proteins and plasma membranes, the surface interaction becomes more active. ${ }^{6,7)}$ Besides those parameters, recently, particle morphology was also considered to affect biological behaviors of materials. Metal hydroxide nanomaterials having different morphologies were known to show different sub-cellular compartment when they are taken up by cells. ${ }^{8)}$

In this context, we hypothesized that the morphology of calcite particles can also affect their biological behaviors. However, there has been less interest on morphology effect of calcite particles. In this paper, we prepared calcites having controlled physicochemical properties with different morphologies in order to evaluate morphology effect on biological properties. As physicochemical properties sensitively affect the biological properties, we tried to only change morphology of calcites while other parameters like crystal phase, crystallinity, particle size and surface charge were controlled same. Two morphologies of cubic and spicule were obtained utilizing polyol solvents referred to as 
Sondi et al.'s report. ${ }^{9)}$

Calcite, as biomedical application, is generally administered via oral route, and thus we evaluated their biological behaviors in two kinds of simulated fluids, gastric and intestinal conditions. We mainly demonstrated the biological behaviors of morphology controlled calcites in simulated biological conditions in terms of $\mathrm{Ca}^{2+}$ dissolution, change in surface charge and interaction between calcites and proteins existing in biological environments. Furthermore, we observed the surface of calcites after exposed to biological conditions utilizing electron microscopy in order to study the morphology dependent surface interactions between calcite particles and biological substances. Consequently, we could suggest that the calcite particles behave differently according to their morphologies.

\section{Experimental}

\subsection{Synthesis of calcites and characterization}

In order to prepare calcites with different particle morphologies, two different polyol solvents, such as diethylene glycol (DEG) and tetraethylene glycol (TEG) were utilized as previously reported..$^{9), 10)}$ Typically, $0.25 \mathrm{M} \mathrm{CaCl}_{2} \cdot 2 \mathrm{H}_{2} \mathrm{O}$ and $0.75 \mathrm{M}$ urea were reacted at $433 \mathrm{~K}$ under polyol solvent condition. In order to obtain cubic morphology, reactants were dissolved in DEG and reacted for $1 \mathrm{~h}$. Spicule shaped calcite was prepared in TEG solvent for $3 \mathrm{~h}$. After the designated reaction time, the white precipitates was collected by centrifugation and thoroughly washed with solvent. Each sample was denoted as Cal-C and Cal-S for cubic and spicule shape, respectively.

In order to evaluate crystal phase of obtained calcites, powder X-ray diffraction patterns were recorded by a Bruker AXS D2 phaser diffractometer with $\mathrm{Ni}$-filtered $\mathrm{Cu}-\mathrm{K} \alpha$ radiation $(\lambda=$ $1.5418 \AA$ ). Size and morphology of calcites were visualized with scanning electron microscopy (SEM: FEI QUANTA FEG250). Colloidal properties such as zeta potential and hydrodynamic size were obtained with ELSZ-1000 (Otsuka Electronics Co. Ltd.) after properly dispersed and diluted in deionized water.

\subsection{Preparation of three different simulated body fluids}

We prepared two simulated body fluids, gastric and intestinal condition, considering the general oral administration route of calcite materials. For gastric condition, $0.034 \mathrm{M} \mathrm{NaCl}$ and pepsin $(3.2 \mathrm{~g})$ was dissolved in deionized water. Then $1 \mathrm{M}$ of hydrochloric acid was titrated until $\mathrm{pH} 1.5$ and finally the solution was massed up to $1 \mathrm{~L}^{11), 12)}$ In order to prepare simulated intestinal fluid, bile salt $(87.5 \mathrm{mg})$ and pancreatin $(25 \mathrm{mg})$ were dissolved in simulated gastric fluid and a few drops of $1.12 \mathrm{M}$ sodium bicarbonate solution were added until $\mathrm{pH} 6.8 .^{11)}$

\subsection{Biological behavior evaluation}

To evaluate biological behaviors of calcites with different morphology, each synthetic sample were dispersed in simulated body fluids with concentration of $10 \mathrm{mg} / 1 \mathrm{~mL}$. We collected aliquots $(4 \mathrm{~mL})$ at $15,30 \mathrm{~min}$, and $1 \mathrm{~h}$ from $15 \mathrm{~mL}$ of gastric/ calcite suspension. For intestinal condition, aliquots were collected similarly at $30 \mathrm{~min}, 1$ and $4 \mathrm{~h}$. The sampling time was decided considering digestive retention time in rats. The suspensions were gently stirred during the whole experiments.

In order to evaluate biological behaviors of calcites in simulated body fluid, collected suspension was first subjected to several measurements such as $\mathrm{pH}$ change, zeta potential and hydrodynamic size utilizing $\mathrm{pH}$ meter ( $\mathrm{pH}$ Meter EcoMet-P15) and ELSZ-1000 (Otsuka Electronics Co. Ltd.), respectively. We also carried out protein fluorescence quenching assay with collected suspension in order to evaluate interactions between calcites and plasma proteins in terms of agglomeration and surface adsorption of proteins. ${ }^{13)}$ The fluorescence emission intensity at excitation wavelength of $320 \mathrm{~nm}$ was measured utilizing luminescence spectrometer (Perkin Elmer LS55). The quenching ratio was calculated as a ratio $\left(I_{0}-I\right) / I_{0}$, where $I_{0}$ and $I$ stood for fluorescence emission intensity for negative control and calcite treated samples. Then the suspensions were separated into precipitates and supernatant utilizing centrifugation. The precipitates were dried in vacuum and subjected to XRD and SEM measurement. Supernatant was filtered with syringe filter and $\mathrm{Ca}^{2+}$ dissolution was measured with inductively coupled plasma atomic emission spectroscopy (ICP-AES: Perkin Elmer OPTIMA 7300DV).

\section{Result and discussion}

\subsection{Structural characterizations}

Crystal phase and crystallinity of both Cal-C and Cal-S were evaluated with powder X-ray diffractometer. As shown in Fig. 1, both samples showed highly crystallized calcite structure (JCPDS \# 47-1743) exhibiting (012), (104), (006), (110), (113), (202), (018), (116), (211), (122), (214) and (300) reflections at 23.1, $29.4,31.2,36.1,39.5,43.3,47.3,48.5,56.7,57.7,60.8$ and $64.9^{\circ}$ (2-theta). ${ }^{14)}$ Although the XRD patterns of both calcites were similar in terms of peak position and intensities, we calculated crystallinity utilizing Scherrer's equation [Eq. (1)], ${ }^{15)}$ in order to compare crystallinity of two samples in detail. We utilized (104) peak having the highest intensity for Scherrer's equation analysis.

$$
\text { Scherrer's equation } t=\frac{0.9 \cdot \lambda}{B \cdot \cos \theta}
$$

[t: crystal thickness $(\AA), B$ : full width half maximum of peak (radians), $\theta$ : peak position (radians), $\lambda$ : X-ray wavelength $(\AA)]$

The crystal thickness was calculated 1,150 and $900 \mathrm{~nm}$ for Cal$\mathrm{C}$ and Cal-S suggesting slightly higher crystallinity for cubic shaped calcite. Considering that calcite crystal had trigonal unit cell, it would be favorable for cubic shape to have ordered crystalline phase.

The particle size and morphology of both calcites were visualized with FE-SEM (Fig. 2). Cal-C was determined to have homogeneous distribution of cubic morphology with approximate primary particle size of $915 \mathrm{~nm}$ (standard deviation of 180 $\mathrm{nm}$ ). SEM image of Cal-S showed completely different aspect compared with $\mathrm{Cal}-\mathrm{C}$, exhibiting irregular shaped particles. The magnified image in the inset of Fig. 2 represented that one particle of Cal-S was composed of several triangular spicules. The primary size of each spicule was determined to be approximately $1,270 \mathrm{~nm}$ (standard deviation of $200 \mathrm{~nm}$ ). Different morphology

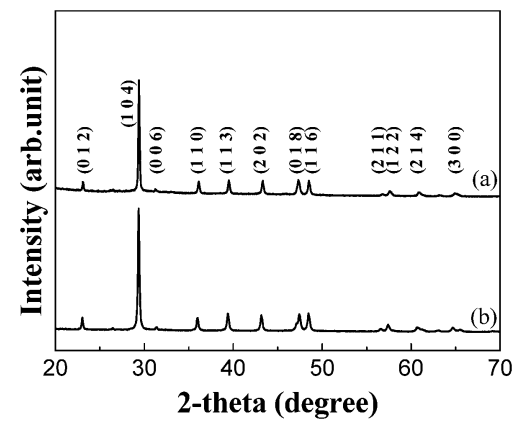

Fig. 1. X-ray diffraction patterns for as-prepared (a) Cal-C and (b) Cal-S. 
of calcite could be explained by the influence of polyol solvent on precipitation growth mechanism of calcite. Sondi et al. pointed out that the adsorption of $-\mathrm{OH}$ groups in polyols on the initial nuclei surfaces inhibited the crystal growth consequently affecting the shape of calcite. ${ }^{9)}$ The cubic and spicule shapes of current calcite particles were well corresponding to Sondi et al.'s results.

The primary particle size obtained from SEM image was comparable with crystal thickness calculated from Scherrer's equation, suggesting that one particle might be composed of one crystal of calcite. It was notable that there was a slight discrepancy between the particle size (SEM result) and crystal thickness (XRD) according to morphology. Crystal thickness of Cal-S was smaller than that of Cal-C in spite of larger primary particle size of Cal-S. It was attributed to the less-favored crystal growth in Cal-S resulted from the mismatch between particle shape and lattice system. The relatively rough surface of Cal-S compared with Cal-C (insets in Fig. 2) corroborated that the crystal growth of Cal-S was less favored than $\mathrm{Cal}-\mathrm{C}$.

The hydrodynamic size and zeta potential of both calcites were summarized in Table 1 with other major physico-chemical properties. Hydrodynamic sizes of Cal-C and Cal-S were determined to be $\sim 2,350 \pm 20$ and $\sim 2,380 \pm 250 \mathrm{~nm}$, respectively, which implied the possible formation of agglomerates of two or three calcite particles in aqueous suspension. Zeta potential values of both calcites in neutral $\mathrm{pH}(\sim 7)$ were slightly negative, which was in good agreement with reference values. ${ }^{16), 17)}$ As the absolute values of zeta potential were less than $10 \mathrm{mV}$, the particles might not be stable in colloid. This resulted in the formation of
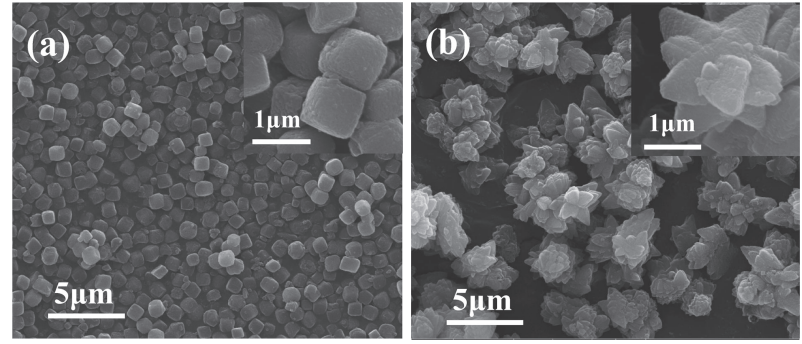

Fig. 2. SEM images for as-prepared (a) Cal-C and (b) Cal-S. agglomeration which could explain the higher hydrodynamic size of calcites compared with the primary particle size obtained from SEM. According to Table 1, both calcites possessed comparable physico-chemical properties in terms of crystal phase, primary particle size, hydrodynamic size and zeta potential, whereas the morphology was significantly different. Therefore we could suggest $\mathrm{Cal}-\mathrm{C}$ and $\mathrm{Cal}-\mathrm{S}$ as standardized calcite samples which were suitable to evaluate morphology effect in biological behavior of calcites.

\subsection{Biological behaviors of calcites}

It was known that calcium absorption in digestive system was mediated with the dissolution of calcium, as calcium was taken up in $\mathrm{Ca}^{2+}$ ionic form mainly in duodenum. Thus we first checked the time-dependent dissolution rate of Cal-C and Cal-S in simulated body fluid conditions. As shown in Fig. 3(a), approximately 3 and $2 \%$ of Cal-C and Cal-S, respectively, dissolved in gastric condition. Both curves readily reached plateau at early stage of $15 \mathrm{~min}$. The difference in dissolution between Cal-C and Cal-S could be influenced by complex factors like crystallinity, particle size, morphology, specific surface area and etc. The specific surface areas measured by $\mathrm{N}_{2}$ adsorptiondesorption isotherm (data not shown) were 6.8 and $2.2 \mathrm{~m}^{2} / \mathrm{g}$ for $\mathrm{Cal}-\mathrm{C}$ and Cal-S, respectively. Considering that the Cal-C had 3 times larger specific surface area than Cal-S, actual dissolution was more facilitated in Cal-S. Thus we could expect that the surface chemical reaction in physiological solution was more active in Cal-S. The dissolution of calcites in intestinal condition was much lower than that in gastric condition [Fig. 3(b)], reaching saturated dissolution of less than $0.15 \%$. The $\mathrm{pH} 6.8$ of intestinal condition was not enough to dissolve calcite to $\mathrm{Ca}^{2+}$ ions, resulting in insignificant dissolution.

Changes in $\mathrm{pH}$, zeta potential as well as protein quenching ratio of differently shaped calcites in simulated body fluid were displayed in Fig. 4. Both Cal-C and Cal-S induced $\mathrm{pH}$ increasing effect due to their highly basic property [Figs. 4(a) and 4(b), respectively]. The $\mathrm{pH}$ value of simulated gastric fluid increased from 1.5 to 7.5 in $1 \mathrm{~h}$, regardless of particle morphology, which strongly related to the prompt dissolution of calcites [Fig. 3(a)]. In case of intestinal condition, the $\mathrm{pH}$ difference was much smaller compared with gastric condition, which was related to the

Table 1. Physico-chemical properties of as-prepared calcites

\begin{tabular}{cccccc}
\hline & Morphology & Crystal structure & $\begin{array}{c}\text { Primary particle size } \\
(\mathrm{nm})\end{array}$ & $\begin{array}{c}\text { Hydrodynamic size } \\
(\mathrm{nm})\end{array}$ & $\begin{array}{c}\text { Zeta potential at } \mathrm{pH} 7 \\
(\mathrm{mV})\end{array}$ \\
\hline Cal-C & Cubic & Calcite & $915 \pm 180$ & $2350 \pm 20$ & $-3.61 \pm 0.67$ \\
Cal-S & Spicule & Calcite & $1270 \pm 20$ & $2380 \pm 250$ & $-6.51 \pm 0.92$ \\
\hline
\end{tabular}
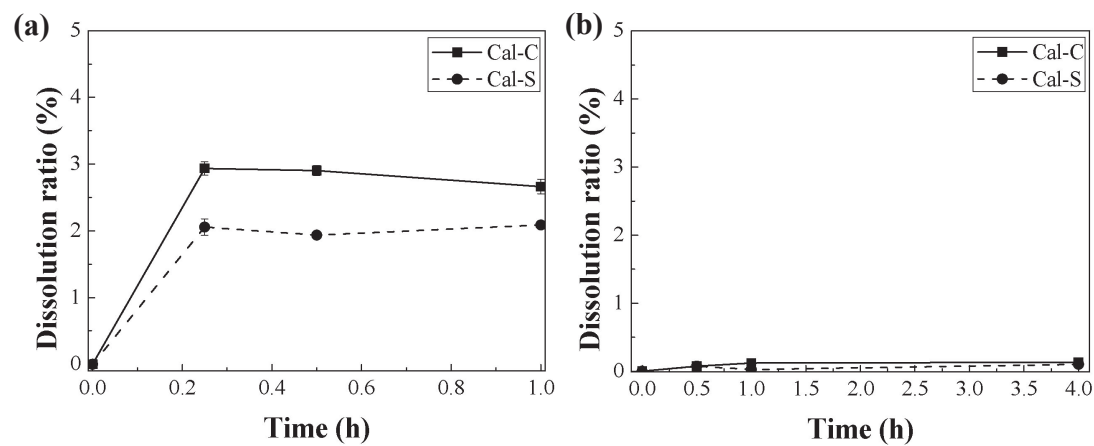

Fig. 3. Time dependent $\mathrm{Ca}^{2+}$ dissolution of Cal-C and Cal-S in simulated (a) gastric and (b) intestinal condition. 
(a)

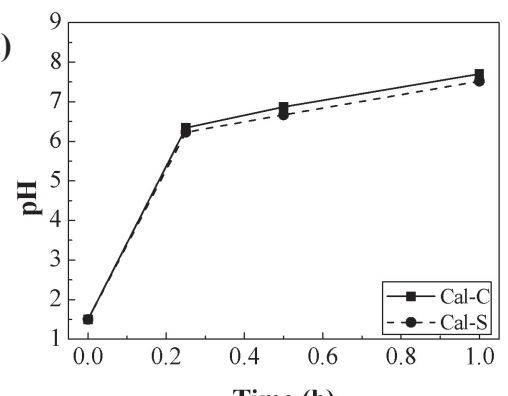

(c)

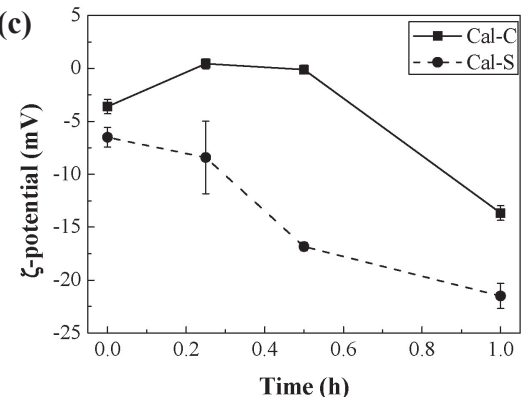

(e)

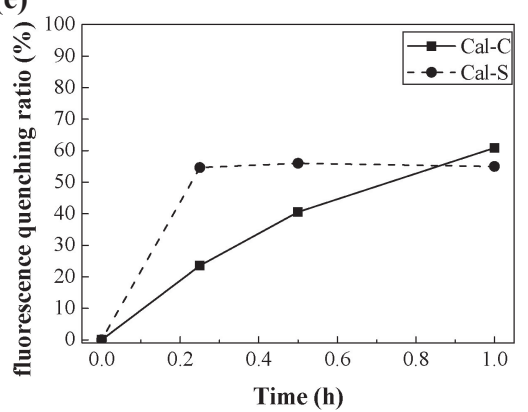

(b)

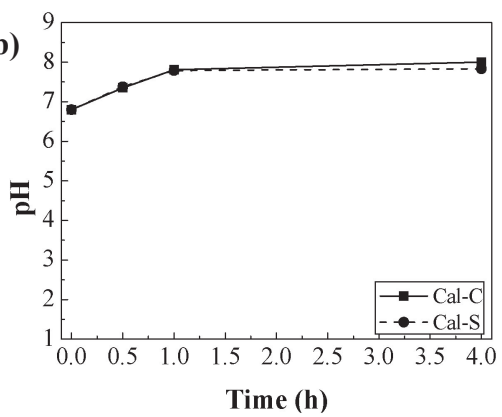

(d)

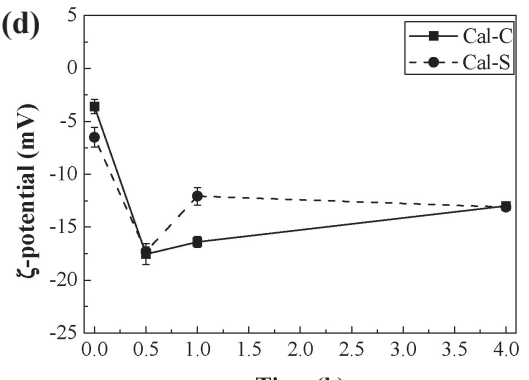

(f)

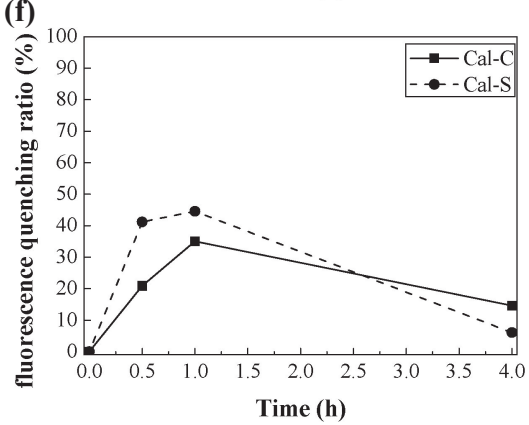

Fig. 4. Time dependent $\mathrm{pH}$ changes of Cal-C and Cal-S in simulated (a) gastric and (b) intestinal conditions. Time dependent zeta potential values of Cal-C and Cal-S in simulated (c) gastric and (d) intestinal conditions. Time dependent and protein fluorescence quenching ratio of Cal-C and Cal-S in simulated (e) gastric and (f) intestinal conditions.

small dissolution.

Zeta potential value changes of calcite particles in simulated gastric fluid exhibited clear difference according to particle morphology. Zeta potential of Cal-C slightly shifted to positive direction due to the $\mathrm{pH}$ lowering and then moved to negative value until $\sim-15 \mathrm{mV}$ in $1 \mathrm{~h}$ [Fig. 4(c)]. On the other hand, Cal-S showed continuously decreasing zeta potential values with respect to time finally reaching zeta potential of $\sim-22 \mathrm{mV}$ [Fig. 4(c)]. This result strongly suggested that the interaction between calcite particles and biological substances in simulated gastric condition such as electrolytes and pepsin was more facilitated in spicule shaped calcite. As the isoelectric point of pepsin was 2.8, it had negative charge in calcite-treated gastric condition $(\mathrm{pH}=6-7)$ and thus the adsorption of pepsin on calcite surface would lower the zeta potential value. The facilitated interaction between proteins and Cal-S compared with Cal-C was also confirmed with protein fluorescence quenching assay [Fig. 4(e)]. Generally, it has been known that the adsorption of protein on particle surface or formation of agglomeration resulted in quenching of fluorescence which was originated from the tryptophan residues in proteins. ${ }^{18), 19)}$ Although both Cal-C and Cal-S reached saturated quenching ratio $\sim 60 \%$ in $1 \mathrm{~h}$ [in gastric condition, Fig. 4(e)], Cal-S showed significantly fast increase in fluorescence quenching ratio. This result also showed that the interaction between Cal-S and biological substances was more active compared with Cal-C. Zeta potential value changes of calcites in simulated intestinal condition was not significantly different according to the particle morphology [Fig. 4(d)]. This might be related to the slight change of $\mathrm{pH}$ value [Fig. 4(b)] and low dissolution rate of calcites [Fig. 3(b)]. However, the protein fluorescence quenching ratio in intestinal condition was different according to particle morphology. Figure 4(f) showed maximum quenching ratio $\sim 45 \%$ of Cal-S compared with $\sim 35 \%$ of Cal-C. In simulated intestinal condition, there existed various biological substances such as pepsin, pancreatin and bile salts, which could actively interact with Cal-S. The relatively small difference of quenching ratio as to particle morphology in intestinal condition [maximum difference $\sim 20 \%$ at $0.5 \mathrm{~h}$, Fig. 4(f)] compared with gastric condition [maximum difference $\sim 40 \%$ at $0.25 \mathrm{~h}$, Fig. 4(e)] might be attributed to the different dissolution rate depending on type of simulated fluid. As shown in Fig. 3, the gastric condition accelerated particle dissolution at the surface of calcites exposing more crystal defects. Thus the surface interaction of calcite with biological substances was more clearly distinguished in gastric condition.

We observed the particle morphology of both calcites treated in simulated body fluids utilizing FE-SEM (Fig. 5). Although the overall particle size and morphology were fairly maintained, the calcites dispersed in simulated body fluids exhibited significant change in terms of surface appearance, compared with the 

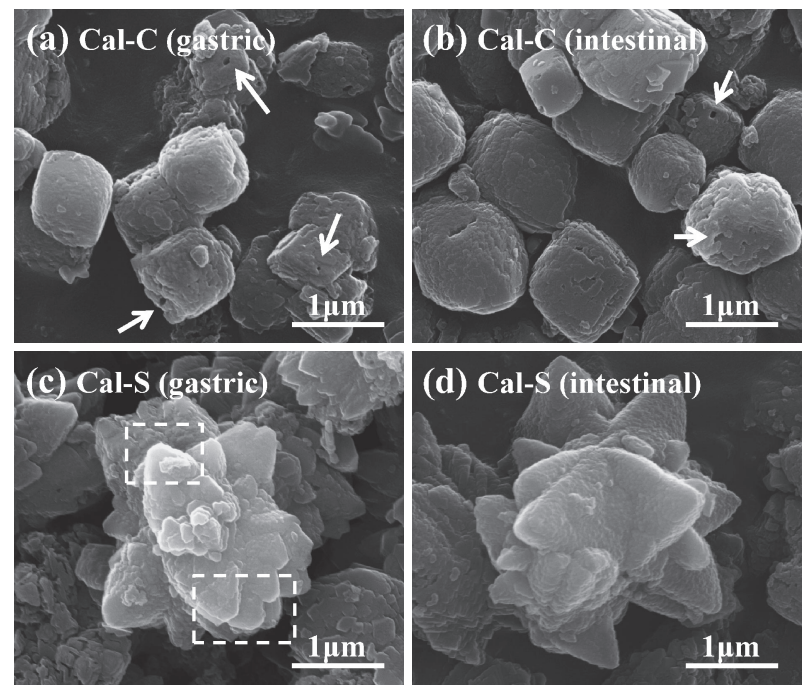

Fig. 5. SEM images for Cal-C treated in (a) simulated gastric condition after $1 \mathrm{~h}$ and (b) simulated intestinal condition after $4 \mathrm{~h}$. Cal-S treated in (c) simulated gastric condition after $1 \mathrm{~h}$ and (d) simulated intestinal condition after $4 \mathrm{~h}$

as-prepared calcites (Fig. 2). This was attributed to the surface dissolution and interaction with biological substances as previously described. We could observe several surface cavities on surface of Cal-C [arrows of Figs. 5(a) and 5(b)], and the formation of cavity was more severe in $\mathrm{Cal}-\mathrm{C}$ in gastric condition than in intestinal condition. Similarly, Cal-S in gastric condition underwent change in surface appearance; the spicule became blunt [dotted rectangles in Fig. 5(c)]. On the other hand, the spicule shape of Cal-S in intestinal condition was fairly maintained. The formation of lumps on Cal-S surface in simulated condition might be due to the surface adsorbed biological substances like electrolytes and proteins.

\section{Conclusion}

We prepared calcite particles with different morphologies (cubic and spicule) utilizing different kinds of polyol solvents. Two kinds of prepared calcite particles, Cal-C and Cal-S, possessed similar physicochemical properties in terms of crystallinity, primary particle size, hydrodynamic size and zeta potential. On the other hand, they showed clearly distinguishable morphology. Thus we utilized those two samples in in vitro biological behavior evaluation in order to find morphology effect of calcite in biological condition. In simulated gastric condition, two types of calcites showed different behavior with more facilitated surface dissolution in Cal-S compared with $\mathrm{Cal}-\mathrm{C}$ considering surface area. Changes in zeta potential value of calcites strongly exhibited the possible interaction between calcite particles and biological substances. Cal-S clearly exhibited zeta potential decrease in simulated condition compared with
Cal-C, suggesting the facilitated surface interaction of spicule shaped calcites. Protein fluorescence quenching assay corroborated the more active surface adsorption of protein on Cal-S than on Cal-C. Morphology dependent biological behavior was more clearly observed in gastric fluid than in intestinal one. As the low $\mathrm{pH}$ in gastric condition induced surface dissolution, which was visualized with SEM, the surface interaction was more clearly distinguished. We could conclude that the morphology was one of factors in calcite, which determined the interaction between calcite particles and biological substances.

Acknowledgement This research was supported by a grant (13182MFDS608) from Ministry of Food and Drug Safety in 2013.

\section{References}

1) Y. Ocak, A. Sofuoglu, F. Tihminlioglu and H. Boke, Prog. Org. Coat., 66, 213-220 (2009).

2) L. Brecevic and D. Kralj, Croat. Chem. Acta, 80, 467-484 (2007).

3) M. G. Tordoff, Physiol. Rev., 81, 1567-1597 (2001).

4) M. S. Jeong, H. S. Cho, S. J. Park, K. S. Song, K. S. Ahn, M.-H. Cho and J. S. Kim, Food Chem. Toxicol., 62, 308-317 (2013).

5) K. Donaldson, P. H. Beswick and P. S. Gilmour, Toxicol. Lett., 88, 293-298 (1996).

6) G. Su, H. Zhou, Q. Mu, Y. Zhang, L. Li, P. Jiao, G. Jiang and B. Yan, J. Phys. Chem. C, 116, 4993-4998 (2012).

7) L. Chen, J. M. McCrate, J. C. M. Lee and H. Li, Nanotechnology, 22, 105708-105717 (2011).

8) Z. P. Xu, M. Niebert, K. Porazik, T. L. Walker, H. M. Cooper, A. P. J. Middelberg, P. P. Gray, P. F. Bartlett and G. Q. Lu, J. Controlled Release, 130, 86-94 (2008).

9) S. D. Škapin and I. Sondi, J. Colloid Interface Sci., 347, 221226 (2010).

10) Q. Li, Y. Ding, F. Li, B. Xie and Y. Qian, J. Cryst. Growth, 236, 357-362 (2002)

11) T.-J. Fu, U. R. Abbott and C. Hatzos, J. Agric. Food Chem., 50, 7154-7160 (2002).

12) J. D. Astwood, J. N. Leach and R. L. Fuchs, Nat. Biotechnol., 14, 1269-1273 (1996).

13) S. H. D. P. Lacerda, J. J. Park, C. Meuse, D. Pristinski, M. L. Becker, A. Karim and J. F. Douglas, ACS Nano, 4, 365-379 (2009).

14) C. G. Kontoyannis and N. V. Vagenas, Analyst, 125, 251-255 (2000).

15) A. L. Patterson, Phys. Rev., 56, 978-982 (1939).

16) E. Chibowski, L. Hotysz and A. Szcześ, Colloids Surf., A, 222, 41-54 (2003).

17) P. Moulin and H. Roques, J. Colloid Interface Sci., 261, 115126 (2003).

18) P. Aggarwal, J. B. Hall, C. B. McLeland, M. A. Dobrovolskaia and S. E. McNeil, Adv. Drug Deliv. Rev., 61, $428-437$ (2009).

19) L. Lai, C. Lin, Z.-Q. Xu, X.-L. Han, F.-F. Tian, P. Mei, D.-W. Li, Y.-S. Ge, F.-L. Jiang, Y.-Z. Zhang and Y. Liu, Spectroc. Acta Pt. A-Molec. Biomolec. Spectr., 97, 366-376 (2012). 